Gazi University
Journal of Science
http://dergipark.gov.tr/gujs

\title{
Advances in Thermal Modeling and Analysis of Satellites
}

\author{
Cihan ATAR*(i), Metin AKTAS \\ Ankara Ylldırım Beyazıt University, Department of Energy Systems Engineering, 06010 Ankara, Turkey
}

Highlights

- This paper focuses on available concepts in thermal modeling and analysis of satellites.

- Both analytical and numerical approaches used in satellite thermal modeling are widely discussed.

- Thermal environment and heat transfer mechanisms in space are described in the paper.

\begin{tabular}{l} 
Article Info \\
\hline \\
Received: 14 Dec 2020 \\
Accepted: 15 Mar 2021 \\
Keywords \\
\hline Satellite \\
Thermal analysis \\
Thermal modeling \\
Analytical approach \\
Numerical method
\end{tabular}

\begin{abstract}
There has been increasing interest in satellite design and manufacture. Satellites require complex components that must withstand severe mechanical and thermal conditions. Thermal design of a satellite relies on several dynamic factors. Satellites experience both extreme temperatures and rapid temperature changes. This situation induces high thermal stresses and may damage components of satellites on-board. In order to build a safely operating satellite for space conditions, this is to be modeled, simulated and tested accurately for several scenarios. Analytical approaches are established to perform thermal analysis in the early stage of satellite technology. Various methods are applied to solve heat balance equations analytically. Though the efforts provide good approximation, more detailed studies are required to perform multi-objective evaluations to determine the optimal design parameters. Numerical procedures play a crucial role for thermal modeling. Commercial software and built-in tools are useful in solving of heat balance equations numerically for achieving temperature distribution of complex and detailed structures. Besides, numerical procedures increase the accuracy and enable flexibility to designers. However, they have drawbacks on excessive calculation times. This paper focuses on available concepts in thermal modeling and analysis of satellites and to demonstrate recent trends in thermal design of satellites. The motivation of this study is to arouse the researchers to pay attention to the basic understanding for the thermal modeling and analysis of satellites under space conditions. In this review, we present useful knowledge about the novel trends and further studies to design, model and analyze the space satellites.
\end{abstract}

\section{INTRODUCTION}

Over the last few decades, developing new technologies and creating new designs in the field of spacecraft have constituted the main frames of research for engineers and scientists. Satellites, artificial objects launched into orbits intentionally, have important roles to improve the state of the world and been performed several different missions for both military and civilian activities such as surveillance, communication and navigation. The design and development of a satellite requires recognizable phases of engineering process. In spacecraft design, accommodating everything including equipment, crew, provisions and payload into the structure must be considered. Satellites generally require lightweight designs. In the aim of satellite designs, optimization processes for both mechanical and thermal strength per weight and efficiency of the design must be considered.

Determination of the thermal characteristics of satellite structures is very crucial step to keep all equipment and the satellite itself functional and operational within the allowable temperature limits for all stages a satellite goes through. Real space thermal tests or even ground simulation tests are very expensive, and they are neither easy nor feasible to perform. Therefore, investigating the thermal behavior of satellites requires 
several mathematical approaches for different satellite configurations, component location, orbital conditions, attitudes and power requirements.

\section{SPACE THERMAL ENVIRONMENT}

Not only the operational lifetime but also the performance of satellites is highly dependent on the influence of thermal environment presented in space. The thermal environment is not stable and changes periodically during the operational period. It also differs with respect to the orbital characteristics. In order to perform thermal modeling of a spacecraft, ambient temperature that a space vehicle experience should be accurately defined. The thermal environment of a vehicle in space includes direct solar, albedo and planetary infrared thermal radiation, as illustrated in Figure 1 [1].

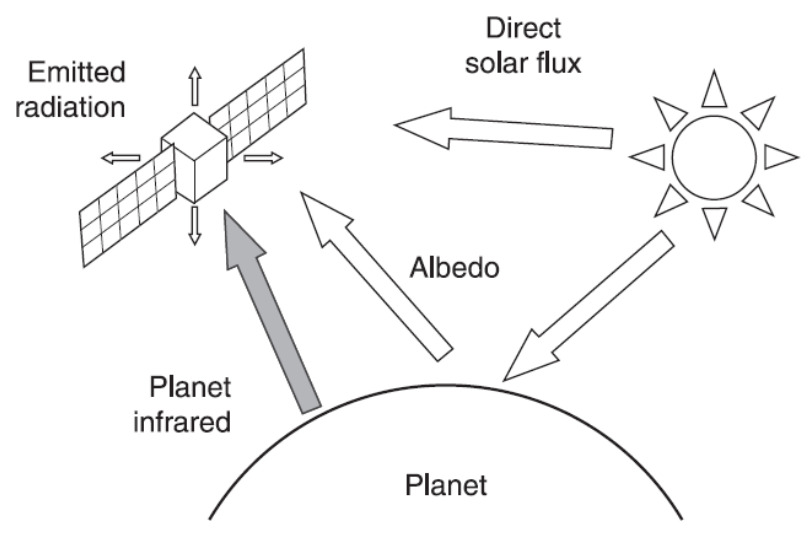

Figure 1. Space thermal environment [1]

In addition, the internal heat dissipation from electronic components mounted on satellite is another heat source to be taken into account.

\subsection{Direct Solar Flux}

The direct solar flux is by far the most intense heat input for a satellite. The spectral distribution of solar irradiation is an important parameter since it directly effects the magnitude of solar flux and the sun can be approximated as a black-body at $5762 \mathrm{~K}$ to simplify the thermal modeling [1]. Depending on the distance to sun the direct solar flux has a mean value of $1367 \mathrm{~W} / \mathrm{m}^{2}$ at $1 \mathrm{AU}(149.5 \mathrm{Mkm})$ and it can be calculated as a function of a distance between a body and the sun in astronomical units as presented in Equation (1) [2]

$\dot{Q}_{\text {solar }}=(1367.5) /(A U)^{2}$.

The heat absorbed by the spacecraft due to the direct solar flux can be analytically written as shown in Equation (2)

$\dot{Q}_{\text {solar }}=\alpha q_{\text {solar }} A_{\text {solar }} \cos \theta$.

It can be understood from the equation that the actual absorbed heat energy depends on the distance from the sun, the thermo-optical properties of the actual surface and the exposed area.

\subsection{Albedo - Reflected Solar Radiation}

Albedo, reflected solar radiation from illuminated side of the planet, is another heat input to a satellite. Albedo radiation is a geometric heating property depending on the position of a satellite and it varies significantly with the orbital altitude. As a result, the influence of Albedo on a space vehicle is higher for low-altitude orbits. The cloud coverage, land and sea areas of planets also have an influence on Albedo. Sea areas reduce the amount of Albedo since they absorb most of the incident radiation. Forest areas also 
have lower Albedo coefficients while the situation is opposite for desert areas. The cloud coverage is another factor altering the local Albedo. Besides, the roughness of a planet's surface changes the reflected solar incident. Albedo values are different for different planets and moons. The average Albedo of the Earth is assumed to be around 30\%. Albedo radiation is only significant for low Earth orbits (LEO) when Earth orbits are considered. Albedo radiation for space vehicles on geostationary orbits (GEO) can be practically neglected. The orbits of satellites traveling around the Earth are shown in Figure 2 [3].

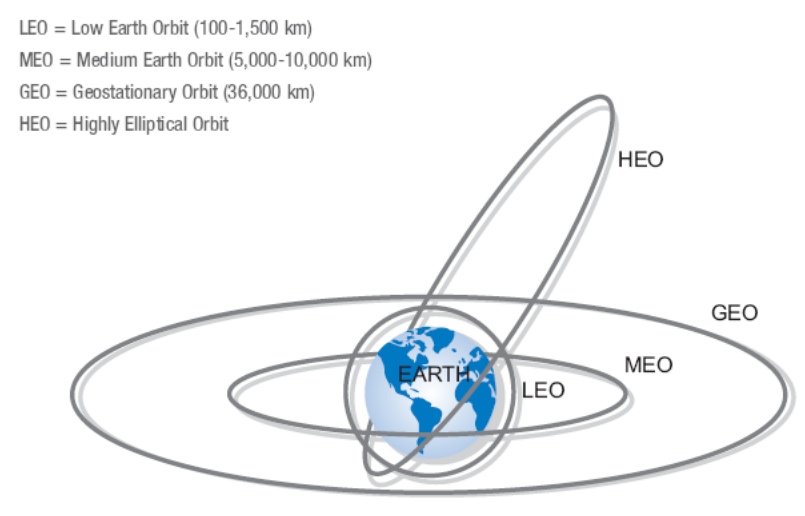

Figure 2. Satellite orbits from the Earth [3]

The heat absorbed by the spacecraft from Albedo can be estimated as in Equation (3)

$\dot{Q}_{\text {Albedo }}=a q_{\text {Albedo }} A_{\text {Albedo }} F_{S / c-p} \cos \phi$.

In that analytical expression, the planet is assumed to behave as a reflecting sphere [1].

\subsection{Planetary-Infrared Radiation}

The third radiant heat input for a satellite is planetary radiation that is also termed as transmitting long wave radiation. That is due to the fact that planets absorb some radiation and reradiate as infrared rays. The planetary radiation also varies from one point to another on the surface of planet because of the different thermo-optic characteristics [1].

The heat absorbed by the spacecraft from planetary-infrared radiation can be estimated as shown in Equation (4)

$\dot{Q}_{\text {planet }}=\varepsilon q_{\text {planet }} A_{\text {planet }} F_{s / c-p}$

Planetary radiation is especially an important heat input for satellites on LEO. The Earth is a heat source of approximately $290 \mathrm{~K}$ providing far infrared heating. Although the quantity of this radiant energy remains more or less constant for circular orbits, it varies significantly for elliptical orbits. For Highly Elliptical Orbit (HEO), on the other hand, the amount of emitted planetary radiation is typically so small that is neglected. There are slight differences in spectral distribution of these infrared radiations due to day and night, latitude, and seasons, however is considered as constant. The Earth for thermal modeling can be assumed as an equivalent black-body transmitting a heat flux of $237 \mathrm{~W} / \mathrm{m}^{2}$ at a mean temperature of 255 K.

\subsection{Internal Heat Dissipation}

The fourth heat input is heat generation from internal electronics mounted in a satellite. This input varies depending on the amount of collected heat energy from external heat sources, the amount of heat converted into electrical energy, the amount of consumed power by the electronics since that eventually results in heat generation. 


\subsection{Extreme Conditions}

Satellites in space environment experience extreme conditions dominated by vacuum existence. So, cooling down of some components on the satellite is very difficult while heating of them is required sometimes depending on the position according to the Sun. The design of the satellite is generally determined by considering periodic solar conditions and the daily changes in both solar maxima and minima. The extreme case scenario corresponds to the situation when one of the sides is receiving the full direct solar radiation and another side of the frame facing the Earth gets the full heat flux of the albedo and Earth infrared energy.

\section{HEAT TRANSFER MECHANISMS}

It is necessary to understand each and every mode of heat transfer when performing thermal analyses of satellites. Heat can be transferred from one place to another by three mechanisms: conduction, convection, and radiation. In satellite thermal analyses, the main mechanisms of heat transfer are thermal radiation exchange and conduction through the materials of the satellite [4]. However, convection that is heat transfer owing to the interaction of a fluid motion over a surface, can be ignored in space because of the existing vacuum condition there.

\subsection{Conduction}

Conduction is a form of heat transfer occurring on account of colliding and diffusing microscopic particles. Heat by conduction can be transferred through any medium, but the rate of thermal conductivity can alter extremely between different materials. Conduction is responsible for most of the heat transferred in a satellite. Thermal management of a spacecraft can be adjusted by selecting materials according to their conductivities and by applying insulators to reduce the rate of conduction.

Conduction heat transfer is governed by Fourier's Law according to the conduction rate, temperature gradient and the direction of energy flow [5]. The differential form of Fourier's Law of thermal conduction can be written as given in Equation (5)

$\vec{q}=-k \nabla \mathrm{T}$.

Hence, the amount of heat transfer via conduction can be described as in Equation (6)

$Q=\frac{A}{L} k\left(T_{h}-T_{c}\right)$

Thermal conductivity over time in 3D space can be written more accurately as shown in Equation (7)

$\frac{\partial}{\partial x}\left(k_{x} \frac{\partial T}{\partial x}\right)+\frac{\partial}{\partial y}\left(k_{y} \frac{\partial T}{\partial y}\right)+\frac{\partial}{\partial z}\left(k_{z} \frac{\partial T}{\partial z}\right)=\rho c \frac{\partial T}{\partial t}$.

This form is called as heat equation and the temperature distribution $\mathrm{T}(x, y, z)$ as a function of time can be obtained from its solution.

\subsection{Radiation}

Radiation is another form of heat transfer by means of travelling particles or electromagnetic waves. An orbiting space vehicle confronts three types of radiation: direct solar flux, albedo, and planetary infrared.

Radiation heat transfer is governed by Stefan-Boltzmann's Law defining the radiation rate according to the absolute temperature of a black-body [5]. The basic equation governing radiation heat transfer can be written as in Equation (8)

$Q=\varepsilon \sigma A\left(T_{1}{ }^{4}-T_{2}{ }^{4}\right)$ 
Radiation heat transfer from direct solar incidents has the highest energy content for a satellite. The amount of radiated heat transfer depends on the satellite's distance from the sun, satellite's direction towards the sun and the solar absorptance to thermal emittance ratio. The greater the temperature difference between two bodies, the greater the heat is transferred via radiation.

\subsection{Energy Balance Equation}

Thermal energy management of a spacecraft is a process that is highly influenced by environmental heating. The major forms of environmental heating during the service of a satellite on orbit are direct solar flux, Albedo, and planetary-infrared energies. Basically, the comprehensive thermal balance of a satellite can be obtained by managing the energy transmitted by the satellite against the energy received from the external environment plus the energy dissipated by the internal electronic components [6]. Therefore, the energy balance equation on the satellite can be written as shown in Equation (9)

$\mathrm{C} \frac{d T}{d t}=\dot{Q}_{\text {in }}-\dot{Q}_{\text {out }}$.

The external loads of direct solar, Albedo and planet-infrared and the internal load of heat dissipation from the electronic equipment are heat inputs to a satellite while the radiated heat from that is output.

$\dot{Q}_{\text {in }}=\dot{Q}_{\text {solar }}+\dot{Q}_{\text {Albedo }}+\dot{Q}_{\text {planet }}+\dot{Q}_{\text {internal }}$.

$\dot{Q}_{\text {out }}=\dot{Q}_{\text {radiated }}$

Hence, the balance equation can be given as in Equation (12)

$\mathrm{C} \frac{d T}{d t}=\dot{Q}_{\text {solar }}+\dot{Q}_{\text {Albedo }}+\dot{Q}_{\text {planet }}+\dot{Q}_{\text {internal }}-\dot{Q}_{\text {radiated }}$

To represent the physical system of a satellite mathematically, a simplified geometry is defined, and this geometry includes only the necessary thermal details. It is called as geometrical mathematical model. After defining the geometry, it is discretized in a network of nodes. The balance equation of each node can be expressed as in Equation (13)

$C_{i} \frac{d T_{i}}{d t}=\dot{Q}_{\text {solar }, i}+\dot{Q}_{\text {Albedo }, i}+\dot{Q}_{\text {plane }, i}+\dot{Q}_{\text {internal }, i}+\sum_{j=1}^{n} K_{i j}\left(T_{j}-T_{i}\right)+\sum_{j=0}^{n} \sigma R_{i j}\left(T_{j}^{4}-T_{i}^{4}\right)$.

Applying the balance equation for all nodes the satellite divided into provides a system of ordinary differential equations and its solution gives the temperature distribution of the satellite. Both two matrices of coefficients for conductive and radiative thermal loads and the vectors for internal and external thermal loads constitute a thermal-mathematical model of a satellite [4]. This formulation also helps to test the thermal balance predictions for all stages of a satellite.

\section{SATELLITE THERMAL DESIGN \& CONTROL}

Satellites include quite complex components that must bear the harsh mechanical and thermal conditions. The satellite structure must have enough strength and stiffness to withstand these severe conditions while it should be lightweight since the cost of launching a satellite is highly depended on its mass. All efforts are, therefore, made to reduce the structural mass of the satellite to the minimum for the same mechanical and thermal performance. This can be achieved by performing a large number of computer simulations and ground tests where similar forces and stresses to be encountered during the lifetime of a satellite are applied [7]. 


\subsection{Satellite Thermal Design}

The thermal design is an activity performed for a satellite to keep all on board components within the desired thermal limits during all stages. The satellite experiences thermal cycles during the entire operational lifetime and the large temperature variations have great influences on the structure and equipment during the eclipse periods. One side facing the sun has extremely high temperatures while the other side facing the colder side of space has extremely low temperatures and the side facing the sun changes periodically when the satellite travelling in its orbit. This situation should be considered in thermal design stage for choosing material and equipment in order to achieve thermal regulation [7].

The satellite thermal design, not only a complex but also an iterative process, is a combination of the design selection and analyzing the selected design. The design selection includes defining the materials, geometries, structural types, allocation of equipment and proper thermal control systems according to the mission criteria of the satellite. The system is analyzed with the selected design parameters to obtain temperature distribution and any change in the design requires new analyses. It is simply driven by the factors of the satellite environment, internal heat dissipation from components, thermal distribution within the satellite, temperature requirements of components and the satellite configuration. An example for the thermal requirements of common satellite components during the service are given in Table 1 [8].

The satellite thermal design should satisfy the requirements to minimize weight, cost, power consumption, and test complexity while providing maximum strength and reliability. This can be achieved by applying the thermal design as simple as possible and avoiding the use of components having moving parts [6]. However, active systems may be needed in some cases and the components have to be chosen according to the mission of a satellite by considering all phases of operation.

Table 1. Typical thermal requirements of components in a satellite [8]

\begin{tabular}{|l|c|c|}
\hline \multirow{2}{*}{ Components } & \multicolumn{2}{|c|}{ Temperature ranges } \\
\cline { 2 - 3 } & $T_{\min }\left[{ }^{\circ} \mathrm{C}\right]$ & $T_{\max }\left[{ }^{\circ} \mathrm{C}\right]$ \\
\hline Electronics (housing) & -10 & +50 \\
\hline Batteries & 0 & +20 \\
\hline Solar arrays & -100 & +120 \\
\hline Antenna dish & -65 & +95 \\
\hline Hydrazine tank & +10 & +50 \\
\hline IR detectors & -223 & -173 \\
\hline Inactive structure & -100 & +100 \\
\hline & Temperature gradients \\
\hline Opto-electronic equipment & \multicolumn{2}{|c|}{$\Delta T<5^{\circ} \mathrm{C}$} \\
\hline High-definition cameras & \multicolumn{2}{|c|}{$\Delta T<0.1^{\circ} \mathrm{C}$} \\
\hline Detectors (CCD) & \multicolumn{2}{|c|}{$\Delta T<0.01^{\circ} \mathrm{C}$} \\
\hline & \multicolumn{2}{|c|}{ Thermal stability } \\
\hline Electronics & $d T / d t<5^{\circ} \mathrm{C} /$ hour \\
\hline Detectors $(\mathrm{CCD})$ in service & \multicolumn{2}{|c|}{$d T<0.1^{\circ} \mathrm{C}$} \\
\hline
\end{tabular}

There are some constraints in satellite thermal design as well. The internal thermal conditions of the satellite should not be affected by the large thermal gradients occur on the external surfaces. The excessive heat generation should be diverted to the colder areas by means of radiation heat transfer mechanism. Since the power generation in the satellite is limited, the active control systems consuming power should not be used or be used only when there is no other solution available for the thermal control. The mass balance and thermal inertia should be sustained for the fixed configuration of on-board components.

\subsection{Thermal Control Systems}

The primary objective of a thermal control system is basically to prevent overheating and undercooling in every part of equipment during the operational lifetime period of spacecraft [9]. There are two types of 
thermal control systems called as active and passive control techniques. Passive control techniques neither have any moving part nor require any energy input while active control techniques need power, sensors and data control from the satellite.

A passive thermal system controls the conductive and the radiative paths to maintain thermal balance and there is no need of power input for thermal regulation within a spacecraft. Passive control can be achieved via the selection of the materials, geometrical configurations, and thermo-optical properties of the surface. This technique is highly advantageous since it provides low cost, volume, weight, and reliability. Passive thermal control techniques used to achieve thermal balance in spacecraft are thermal insulation, thermal coating, heat sinks and phase-change materials [10].

Thermal control may not be obtained adequately and efficiently for applications where a component has a very limited operational temperature range or where a tremendous alteration in heat input during the service exists. In these situations, thermal sensors are installed to the critical locations to be measured and when the critical temperatures are reached, active control systems are engaged to obtain thermal balance. Active techniques rely on input power for operation. The typical active control techniques are heaters, coolers, louvers or the use of the cryogenic materials [10].

\section{THERMAL MODELING \& ANALYSIS}

Thermal analysis of spacecraft has always been one of the most crucial assignments of engineers in the stages of researching, developing, designing, manufacturing, and operating for successful space missions. Thermal design of a spacecraft is a process combining the thermal modeling with the analysis of the results obtained from mathematical models. In satellite analysis, some elemental approaches are required to perform detailed assessments. They are mainly analytical and numerical approaches.

\subsection{Analytical Approaches}

Analytical studies for thermal analysis have been developed and applied in various heat transfer areas. Hence, analytical approach is the first method coming to mind to use for thermal modeling and analysis of satellites. In order to solve heat balance equations analytically for a satellite system, various models are adopted. They include one-node models, two-node models and multi-node models. A linear one-node approximation having only energy transfer with the environment is useful guide during preliminary attempts to spacecraft thermal control whereas it does not offer very accurate predictions. A two-node model is the refined form of the one-node model by adding another node allowing to consider heat transfer between parts. Thermal discretization of spacecraft with more than two or more nodes yields multi-node models providing better approximations.

Oshima and Oshima [11] use analytical methods based on one-node model to face the thermal design problem of a spacecraft; however, the outcomes of their study are not realistic primarily caused by the parameters used and the complexity of the required calculations. Arduini et al. [12] proposed a new approach by means of linearized techniques to solve the inverse problem in satellite thermal control. They use a simple cylindrical structure represented by three nodes. They also state that the pseudo-inverse formulation seems to be a proper tool to solve the problem of correcting of both conduction and radiation coefficients in a mathematical model. However, the comparison of this method with other conventional methods is not included in their study.

A handbook by Gilmore [6] is published for space thermal control applications with the aims of describing the space thermal environments, introducing design approaches and providing thermal control technologies. Not only spacecraft systems and their configurations but their thermal environments as well as their operating orbits are briefly explained. The useful tables of Albedo values, planetary infrared radiation emissions, orbital parameters and environments for different planets are given in the book. He introduces thermal design examples for several parts of satellites as well as their thermal characteristics. Both passive and active thermal control measures used in satellite designs are covered. The finite-difference method 
(FDM) based on Taylor series approximation, the finite-element method (FEM) based on using elements in different dimensions and their comparison are explained.

Tsai [13] reviews general thermal mathematical model and simple thermal analytical models for thermal control of satellite. A brief mathematical background for analytical formulations to solve, confirm and understand the limitations is given in his study. He constructs a system of finite difference equations for thermal mathematical model by means of Taylor series approximation. In order to perform iterative calculations to get solution for the constructed model, he provides explicit, implicit, Crank-Nicolson and Dufort-Frankel schemes. The different cases from the simplest one where there is no coupling for conduction and radiation to the most complicated and realistic one where there are couplings both conduction and radiation are explained. He also introduces linear approximation and exact formulation methods for solving the simplified problem in an analytical way and concludes that both models have equal importance in thermal analysis of satellites since one can offer more accurate results while other can provide faster estimations.

The conventional approach in early period is to simulate spacecraft with geometric primitives. As geometric structure of satellites gains prominence in the view of thermal analysis, studies focuses on different structure types. Ziebart et al. [14] presents a general summary of methodical approaches for examining the nonconservative thermal forces and the complexity in the source radiation fluxes for complex satellite geometries. They apply solar radiation pressure modeling, anisotropic thermal re-radiation force modeling and Albedo/Earthshine force modeling to deal with complex structure. However, their study is mainly focused on orbit determination rather than the thermal modeling.

The thermal analysis of satellites for dynamic systems is another research field and the spinning satellites are most popular cases among them. It is important to simulate rotating conditions since the temperature peaks can be minimized by the effects of spinning. The temperature distribution prediction of a cylindrical, thick-walled space vehicle that is revolving with a constant angular velocity in space and subjected to uniform solar radiation in deep space is investigated by an analytical approximation in Gadalla's study [15]. A complex form of Fourier series is used to obtain the temperature distribution on the surface of the spacecraft by considering both radiative and conductive heat transfer through vehicle wall. He concludes that the symmetry of surface temperature distribution is diminished, and the peak temperature locations are shifted along the angular position for a rotating vehicle. Prajapati et al. [16] study the temperature profile of a spinning satellite by applying fractional calculus approach. The formulation of Caputo fractional model is solved by Laplace transformation to obtain the non-dimensional temperature variation with respect to the spinning rate. Chung et al. [17] perform numerical simulations for a two-node thermal model of small satellite by using dual equivalent linearization technique with Newton-Raphson iteration method. They suggest that their two-node model is available for small compact spinning satellites. They also come up with the outcome expressing further extensions to many-node models which are required to study effectiveness and accuracy. A simplified analytical approach for examining the thermal performance of a spinning satellite orbiting the Earth over sun-synchronous orbits is conducted by Farahhi and Perez-Grande [18]. They investigate the effect of spinning on temperature distribution along the satellite structure. They state that the proposed simplified model can be handy for early design stage.

Spacecraft's solar panels have considerable effects on satellite thermal design; therefore, it is important to understand thermal mechanisms of a satellite equipped with solar panels. In order to investigate the thermal behavior and stability of small compact satellites with mounted solar panels is introduced by Perez-Grande et al. [19] by employing a simple analytical approach and they apply Fourier analysis method with an approach having integration of the energy balance equations of a two-node reduced model in order to achieve a linearized second-order ODE problem. Using a simplified mathematical model with non-linear analytical methods, Gaite et al. [20] analyze a simple model of temperature evolution in a satellite by considering the satellite isothermal. Gaite presents another nonlinear thermal analysis of a satellite by considering two-node models and performs the analysis by means of analytical techniques [21].

Sizes of satellites get smaller day-by-day to reduce the launching costs, the development times and to provide relative simplicity and more flexibility although small satellites have serious structural challenges 
because of thermal and power constraints. Since small satellites have limited mass and volume, the thermal modeling becomes very important tool to make sure that space mission is safe and successful. As a result, thermal modeling of small satellites attracts attentions. Baturkin [22] states general thermal control concepts and components for micro-satellite and includes some details on thermal hardware and ground testing of thermal control design as well. Das et al. [23] analyzed a cubic micro-satellite with deployable solar panels by applying two-nodal and three-nodal parametric analyses to obtain the optical properties. The analytical investigation conducted by Bulut and Sözbir [24] for a nanosatellite in LEO is presented to understand the thermal performance of the design for different panel combinations and they deduce that heaters are required to raise the temperature of batteries for cold case scenario.

\subsection{Numerical Approaches}

Thermal analysis of spacecraft and space structures, however, requires different skills because of the fact that non-linearity and complexity exist by its nature. Recently, software tools have been developed to solve the heat balance equations especially for complex and detailed structures. These tools provide more reliable and efficient solutions for very detailed calculations and enable to simulate spacecraft for different designs and service conditions.

The numerical methods available for solving ordinary differential equations of spacecraft thermal problems are summarized and the lumped-mass scheme ordinarily applied in thermal simulations is introduced by Krishnaprakas [25]. A system of first-order, coupled, nonlinear ordinary differential equations (ODEs) for thermal energy balance expression is introduced besides with the solution methods. He also compares these methods and comes up with that Crank-Nicholson method is found to efficiently solve ordinary ODEs associated with the spacecraft thermal mathematical model based on lumped-parameter network. Computer codes not only for solving ODEs but also for analyzing spacecraft heat transfer problems are provided.

In order to simulate the operational thermal behavior of FalconSat-2, a thermal model of the satellite is created in Simulink, a MATLAB-based block diagram for graphical system simulations, by Lyon et al.[26] employing finite difference and lumped-parameter model in the design stage. They seek if any spacecraft component exceeds its temperature limits for on-orbit scenarios and evaluate temperature fluctuations of all components to minimize them. The thermal simulations are conducted for both on-orbit conditions and extreme cases for several attitude and subsystem operation modes. Thermal vacuum tests of FASTRAC, a small satellite, are conducted by Diaz-Aguado et al. [27] and computational thermal analysis by using MATLAB is performed and the thermal behaviors of the satellite for several orbits are simulated by ABAQUS to reveal the readiness for spaceflight. Another study on thermal design and on-orbit thermal performance of a small satellite is carried by Narayana and Reddy [28]. After one-year of service in space, they analyze the HAMSAT, a cuboid micro-satellite traveling at sun-synchronous orbit for providing satellite based amateur radio services, by using a thermal model based on isothermal node concept and the mathematical model is created on NX I-deas TMG Thermal Analysis software. The adequacy of thermal control system of the satellite is investigated for service conditions and it is concluded that all subsystems stay in the designated temperature limits throughout the mission period. Sözbir et al. [29] perform thermal simulations of TUSAT, a Turkish communication satellite operating in GEO, by using ThermXL software to understand the effectiveness of thermal control subsystem. Their study basically states the process of thermal design at the level of preliminary design review stage. They investigate the performance of active and passive thermal control measures to ensure that the satellite operates between the desired temperature limits during its lifetime. Arroyo [5] in his thesis performs orbital and thermal analyses of the UPC CubeSat by means of STK software. The results obtained from his study show similarities with previous projects. Thermal analyses are performed for a simplified geometry and his study illustrates that the temperature varies in a great magnitude. So, he underlines the importance of conducting thermal analyses for detailed geometries since the shape of the satellite governs the important characteristics of the simulation and recommends performing thermal analysis by using available software such as ESATAN, ESARAD or ANSYS. A nanosatellite travelling in LEOs is simulated by Bulut and Sözbir [30] on ThermXL software with a simplified model to obtain rapid solutions. They simulate the satellite by covering all faces with solar panels. They find out that the surface directed towards the sun is the hottest while the one directed to cold space is the coldest and the temperatures on solar cells and the structures are nearly equal. They aim to 
check and compare the results with a detailed analysis on ESATAN software in future. Dinh [31] investigates the thermal performance of a nanosatellite in the LEO for different altitudes. The simulations are carried out with the 3-D model of the Pumpkin CubeSat on Thermal Desktop for external analysis and ANSYS Icepack for internal component and he also concludes that the optimum design can be achieved by updating the thermal model as the modifications in design take place. The study of Garzon [32] is to model the thermal environment for OSIRIS-3U for worst case orbital scenarios. She performs thermal simulations via FEA thermal models developed in COMSOL Multiphysics software. Athirah et al. [33] conduct stress and thermal simulations of a CubeSat by ANSYS in order to analyze safety factor, weight and battery discharge rate. They analyze two structures with and without beam. Thermal simulations in their study are to evaluate the discharge rate of battery when the satellite experiences eclipse conditions and the results show that the internal temperature of the satellite decreases as the discharge period increases. They suggest using heater as a thermal control system for eclipse term lasting longer than one-hour. Silva et al. [34] present thermal control design and analysis of Amazonia-1, a Brazilian remote sensing satellite by applying passive means and heats. The thermal model is developed based on a lumped parameter method assuming the nodes that are isothermal and the simulations are performed by using Thermal Desktop, RadCAD and SINDA/FLUINT that are software packages of C\&R Technologies. They mainly investigate temperature variations and power consumptions of heaters under critical cases. Another thermal modeling of a picosatellite is performed by Wachche et al. [35] and they simulate Swayam, a 1U, LEO satellite using NX Space Systems Thermal software. Several thermal insulation combinations proposed to ensure the safety of satellite components are analyzed in their simulations. The thermal models for PICSAT and CIRCUS nanosatellites are created using Systema-Thermica software and simulations are carried out by Flecht [36]. He first analyzes PICSAT for extreme conditions and for relevant failure modes including the malfunctions of attitude control system and the deployable solar panels. Different configurations of deployable solar panels for CIRCUS are investigated by adjusting the thermal model of PICSAT to get first estimations regarding the thermal performance of the spacecraft in the early design stage. Thermal simulations using Systema-Thermica are performed to analyze the MIST CubeSat by Chandrashekar [37] and he investigates the thermal behavior of the entire satellite for non-operational cold case, operational cold case and operational case during the development stage of the satellite. In order to conduct more realistic simulations for the MIST CubeSat, Bjönberg and Larsson [38] refined the geometrical model and updated the satellite thermal model by adding internal heat dissipation in their study. They suggest further investigations after improving the satellite model according to the results from the study since the simulations performed using Systema-Thermica software show that the current configuration is not safe to serve in space. Similarly, Lahrichi [39] performs a heat analysis of MaSat-1, a cubesat project, using ANSYS software and investigates the heat flows for different orbital conditions. He recommends considering the effects of fatigue and electromagnetic interferences in thermal simulations. A satellite travelling in a sun synchronous orbit around the Earth is also simulated using Systema-Thermica for the worst thermal cases of the orbit by Still [40] and he studies the thermal performance of radiators and heaters of the satellite. The EIRSAT-1, a $1 \mathrm{U}$ sized cubesat, is simulated by Wallace et al. [41] using Thermal Desktop software for the steady analysis. They investigate the thermal responses of the satellite for different orbital conditions and study the thermal performance of solar paints and insulations.

Although solving energy-balance equations by means of commercial software packages provides quick and reliable solutions, there are some efforts not only on analytical approaches but also on combining them with software using coding. Deiml et al. [42] present a study on thermal model reduction algorithms by investigating different linear and nonlinear model reduction methods. They find out that swarm and pattern search algorithms provide convenient results to optimize optical properties while polygon reduction method is useless for reduction of geometric models. To reduce the mathematical models, summation technique can offer sufficient result and it can be compatible with commercial software. Discretizing analytical equations by finite difference approach, thermal analysis of a nanosatellite is performed numerically by Corpino et al. [43] in MATLAB environment in order to investigate passive measures of satellite structure. They create numerical codes in MATLAB to adapt the thermal model to the nanoscale, three-dimesional and time-dependent environment. The outcome from their study is that applying thermal control measures such as coatings and heat sinks can improve thermal management while basic heaters are adequate to keep the temperature between the desired limits during eclipse periods. Equivalent linearization technique is used by Anh et al. [44] for a single-node thermal model of satellites in LEO to obtain linearization 
coefficients and to estimate temperature evolutions for the overall system. Even though the dual criterion of equivalent linearization offers smaller errors when nonlinearity rises in the system, the effectiveness and the accuracy of the method should be investigated with many-node extended studies.

Satellite studies keep focused on both analytical and computational techniques for small satellites. Thermal control of TURKSAT 3U nanosatellite is investigated by Bulut et al. [45] to ensure that all components of satellite operate within their operating temperature ranges. They suggest the application of coating for early stage of design and use of heaters to keep the cube satellite warm for the cold case conditions. For analyzing both structural design and mission requirements of Surya Satellite-1, Steven et al. [46] conduct numerical simulations to obtain natural frequency mode, maximum deformation value, and maximum von Misses pressure by using ANSYS software. In their study, the thermal influence is only considered to perform structural analysis and to understand the structural strength. Another study on small satellite is the thermal analysis performed by Kovacs and Jozsa [47] and they investigate the SMOG-1 PocketQube satellite to reach the same goal of previous works. However, having quite smaller internal volume differs this one from the rest. The simulations are carried out by a thermal network model developed in MATLAB Simulink and by a FEM model in ANSYS Workbench as well. The accuracy of the thermal network model having twelve nodes is poor because of the simplicity it has. Nevertheless, it predicts temperature variations properly. The FEM model, on the other hand, offers higher accuracy while it requires more time to get results due to the large number of nodes. In order to analyze on-orbit thermal behavior of the UoMBSat-1, a pico-satellite, an analytical tool is developed in the study of Bonnici et al. [48] by simplifying the thermal model and applying a lumped parameter approach with a set of seventeen nodes. They also perform numerical simulations on ANSYS and then the results from both approaches are compared. They state that the results from two approaches are agreed. Their conclusion is that maximum temperatures can be controlled by passive measure; however, it is very difficult to handle minimum temperatures due to the limited space capacity causing almost impossible to implement active thermal control measures. The thermal analyses are also performed for post-mission phases to validate early studies and to review the performance of the thermal control systems. The ESEO satellite having a cuboid shape with six structural panels and three solar panels is modeled and simulated by Ahmed and Ahmed [49] using Thermal Desktop software. They investigate the temperature variations of components and solar panels as well. The satellite thermal model is verified by changing design variables and checking the temperature values.

\subsection{Thermal Simulation Procedure}

Thermal simulation of a satellite starts with creating the satellite geometry based on the design configuration and building the mathematical representation of that geometry. The next step is discretizing the geometry in a network of nodes by meshing. The balance equation is applied to all nodes and then both the external and the internal loads are defined. The equation is solved for each node to get temperature distribution by applying different mathematical techniques and can be solved for extreme conditions to understand the upper and lower temperature limits for the space mission. As long as there are no strict stability requirements, the thermal analysis is obtained from steady calculations. Transient calculations, on the other hand, are performed to determine temperature variations by time once the steady-state solutions are obtained [4]. The balance equations for both steady-state and transient conditions can be difficult to solve with conventional methods so that the simulation software tools are required to get solutions.

\subsection{Simulation Software Tools}

The main objective of all thermal-analysis studies is solving the general energy balance equation. Applying the balance equation to the spacecraft system including all subsystems can be challenging and timeconsuming with the analytical approaches. As a result, thermal analysis software tools have been developed based on numerical description of the satellite to simulate complex physical thermal networks. These tools have model generator, solver and post-processor to display temperature distribution and heat flux plots. Some of them have an ability to read computer-aided designs created in other computer programs and to process them. Thermal analyzers are mainly based on lumped-parameter, finite-difference or finite-element schemes while radiation codes are based on the gray-diffuse assumption or Monte-Carlo approach [6]. 
There are several commercial software tools available in the aerospace industry and they have different capabilities, as compared in Table 2. TRASYS, Thermal Radiation Analyzer System, is a software developed by NASA in early 1970s to perform radiation analysis. Even though the software survives today, use of it has been decreased dramatically due to the limited accessibility and availability of more advanced tools [50]. SINDA/FLUINT, The System Improved Numerical Differencing Analyzer/Fluid Integrator, is used for heat transfer and fluid flow analysis. The model for this tool can be created by using a twodimensional schematic sketchpad called SINAPS or a three-dimensional computer-aided model can be built on Thermal Desktop. It has another module called RadCAD for estimating radiation exchange factors within the thermal model and the environment using the Monte-Carlo ray tracing approach. This tool can use user-defined FORTRAN codes and be linked to MATLAB to make further adjustments in simulations.

The TSS, Thermal Synthesizer System, is another tool developed by NASA to replace TRASYS and to improve older codes. TSS, implemented in C language, can execute thermal simulations for various purposes and offers both internal and external design options to determine temperatures of the spacecraft components [51]. Thermica is a comprehensive spacecraft-oriented modeling tool and performs a complete thermal analysis of an object in its environment.

The main software for spacecraft thermal simulations in Europe is ESATAN-TMS. The radiative and conductive heat transfer calculations can be performed by this tool to find temperatures and heat fluxes over a pre-defined orbit. ESATAN, European Space Agency Thermal Analyzer, is written by FORTRAN language employing thermal network analysis approach and used to predict temperature distributions for spacecraft [4]. ESARAD, European Space Agency Radiation Analyzer, is the main radiation analysis tool developed for ESA by Alstom. It provides an interactive operating mode while offering parametric modeling. It uses Monte-Carlo ray tracing method to estimate view factors, radiative heat exchange factors, direct heat fluxes and absorbed heat fluxes [52]. ESATAN-TMS is an advanced workbench environment for the thermal analysis of spacecraft and launch vehicles while having both ESATAN and ESARAD features. This thermal modeling suite offers useful charts and reports to visualize thermal analyses [53].

Table 2. Comparison of simulation software in terms of their capabilities

\begin{tabular}{|c|c|c|c|c|c|c|}
\hline Capabilities & 点 & 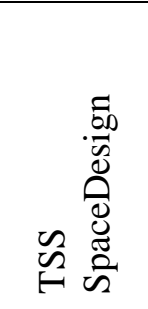 & 点 & 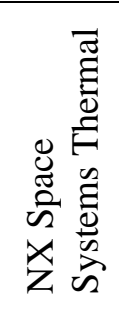 & 它 & $\begin{array}{l}\tilde{\lambda} \\
\bar{Z} \\
z\end{array}$ \\
\hline GMM generator & AutoCAD & Space3D & CADbench & NX & $\begin{array}{l}\text { Design } \\
\text { Module }\end{array}$ & $\begin{array}{c}\text { Discovery } \\
\text { SpaceClaim }\end{array}$ \\
\hline Bulk property definition & $\checkmark$ & $\checkmark$ & $\checkmark$ & $\checkmark$ & $\checkmark$ & $\checkmark$ \\
\hline Optical property definition & $\checkmark$ & $\checkmark$ & $\checkmark$ & $\checkmark$ & $\checkmark$ & $\checkmark$ \\
\hline Shell surface definition & $\checkmark$ & $\checkmark$ & $\checkmark$ & $\checkmark$ & $\checkmark$ & $\checkmark$ \\
\hline Generic meshing & $\checkmark$ & $\checkmark$ & $\checkmark$ & $\checkmark$ & $\checkmark$ & $\checkmark$ \\
\hline Mesh operations & $x$ & $\checkmark$ & $\checkmark$ & $\checkmark$ & $\checkmark$ & $\checkmark$ \\
\hline Defining assemblies & $\checkmark$ & $\checkmark$ & $\checkmark$ & $\checkmark$ & $\checkmark$ & $\checkmark$ \\
\hline Surface boundary conditions & $\checkmark$ & $x$ & $\checkmark$ & $\checkmark$ & $\checkmark$ & $\checkmark$ \\
\hline Radiation analyzer & RadCAD & Radk & ESARAD & Built-in & Built-in & Built-in \\
\hline Thermal analyzer & SINDA & SINDA & ESATAN & Built-in & Built-in & Built-in \\
\hline Coupled thermo-fluid & $\checkmark$ & $\checkmark$ & $\checkmark$ & $\checkmark$ & $\checkmark$ & $\checkmark$ \\
\hline Coupled thermo-mechanic & $x$ & $x$ & $x$ & $\checkmark$ & $\checkmark$ & $\checkmark$ \\
\hline
\end{tabular}

Another important tool used mostly in Europe is NX Space Systems Thermal, formerly known as NX Ideas TMG Thermal Analysis software. It is developed by Maya HTT and a software of Siemens Product Lifecycle Management solutions. It enables to generate three-dimensional geometries and create finite element models. This coded by FORTRAN language offers rapid and accurate thermal simulation for 
nonlinear and transient heat transfer processes. It uses a finite element-based analysis with a finite volume solver. Besides, the tool enables to simulate thermal couplings [54].

Moreover, there are ANSYS and COMSOL Multiphysics software packages for general-purpose multiphysics analyses. COMSOL Multiphysics offers modular simulation based on finite element analysis. It can simulate a wide range of engineering applications and provides modules for different problems to be analyzed. Similarly, ANSYS is a commercial software using finite element methods to perform simulations for engineering problems. ANSYS Workbench, providing all modules in one platform, allows to simulate and analyze coupled systems. Both of them have built-in three-dimensional mode while they can communicate with external drawing and modeling tools.

\section{CONCLUSION}

In this study, a concise background about the thermal environment and heat transfer mechanisms of satellites in space are introduced. The heat exchange between the satellite and its surrounding space mainly occurs by radiation while heat exchange through the materials of the satellite takes place by conduction. Space environmental effects including direct solar radiation, albedo radiation and planetary-infrared radiation vary greatly with respect to the orbital characteristics. Understanding both the heat transfer mechanisms and the environmental effects in space is crucial to facilitate a proper thermal design. Therefore, the affecting thermal loads and the fundamental equations are presented besides with the worstcase definitions.

The satellite thermal design with regarding the processes of design selection and analyzing the selected design shall satisfy the mission requirements. The objectives of thermal design have been described in this study along with the design constraints. Thermal control systems to meet the required operational limits of satellites are also stated by describing both active and passive measures.

The detailed review on available concepts in thermal modeling and analysis of satellites are expressed. There is a substantial interest and development in them to survive and operate in extreme space conditions. In order to understand well the phenomena, analytical and numerical tools can be implemented. Analytical approaches can provide predictions for early stage of the design. To obtain reliable results with them for defined conditions, accurately defining the problem and having good estimations are expected. On the other hand, numerical procedures supply more accurate solutions while requiring longer computing times. It can be noted that investigating the complex and detailed structures of satellites is possible by implementing these approaches. They also offer flexibility to modify designs to re-simulate. Furthermore, analytical techniques can be combined with numerical software to investigate a custom case with specific features entirely and precisely. Additionally, recent trends in thermal design of small satellites are demonstrated since the literature of the thermal design of satellites has been enriching day-by-day and especially the studies on small scale (micro, nano and pico) satellites have been increasing. Finally, thermal simulation procedures and available simulation software tools for satellite thermal modeling are discussed in the study.

To conclude, we hope this paper broadens the knowledge for familiarizing with recent developments in thermal design, modeling and analysis of satellites and may help scholars to encourage for researching in the extent of their thermal modeling and analysis.

\section{CONFLICTS OF INTEREST}

No conflict of interest was declared by the authors.

\section{REFERENCES}

[1] Meseguer, J., Pérez-Grande, I., Sanz-Andrés, A., Spacecraft Thermal Control 2 - Space environment, $15-38,(2012)$. 
[2] Birur, G. C., Siebes, G., Swanson, T. D., Encyclopedia of Physical Science and Technology, 3rd Edition, Spacecraft Thermal Control, New York, 485-505, (2003).

[3] Karim, S., Sakib, S., Islam, M., Ahamed, F. A. S., "A review of communications satellite by focusing on 'Bangabandhu Satellite-1', the first GEO communications satellite of Bangladesh", International Journal of Computer Networks and Communications, 8: 123-128, (2018).

[4] Meseguer, J., Pérez-Grande, I., Sanz-Andrés, A., Spacecraft Thermal Control 19 - Thermal Mathematical Models, 339-348, (2012).

[5] Sintes Arroyo, P., "Mission and thermal analysis of UPC cubesat", MSc. Thesis, Universitat Politecnica de Catalunya Aerospace Science and Technology, Barcelona, Spain, 125, (2009).

[6] Gilmore, D.G., Spacecraft Thermal Control Handbook, Volume 1: Fundamental Technologies, (2002).

[7] Maini, A. K., Agrawal, V., Satellite Technology: Principles and Applications, (2010).

[8] Meseguer, J., Pérez-Grande, I., Sanz-Andrés, A., Spacecraft Thermal Control 18 - Thermal Control Design, 327-338, (2012).

[9] Martinez, I., Spacecraft Thermal Control, Modeling and Testing, Madrid, (2019).

[10] Staff, M. D. D., "Small spacecraft technology - state of the art", NASA/TP-2014-216648/REV1, Moffett Field, California, (2014).

[11] Oshima, K., Oshima, Y., "An analytical approach to the thermal design of spacecrafts", Institute of Space and Aeronautical Science, (1968).

[12] Arduini, C., Laneve, G., Folco, S., "Linearized techniques for solving the inverse problem in the satellite thermal control", Paper IAF 96.I6.03 presented at the 47th International Astronautical Congress, October 7-11, 1996, Beijing, China", Acta Astronautica, 43: 473-479, (1998).

[13] Tsai, J. R., "Overview of satellite thermal analytical model”, Journal of Spacecraft and Rockets, 41: 120-125, (2004).

[14] Ziebart, M., Adhya, S., Sibthorpe, A., Edwards S., Cross P., "Combined radiation pressure and thermal modelling of complex satellites: Algorithms and on-orbit tests", Advances in Space Research, 36: 424430, (2005).

[15] Gadalla, M. A., "Prediction of temperature variation in a rotating spacecraft in space environment", Applied Thermal Engineering, 25: 2379-2397, (2005).

[16] Prajapati, J. C., Kachhia, K. B., Kosta, S. P., "Fractional calculus approach to study temperature distribution within a spinning satellite", Alexandria Engineering Journal, 55: 2345-2350, (2016).

[17] Chung, P. N., Anh, N. D., Hieu, N. N., Manh, D. V., "Extension of dual equivalent linearization to nonlinear analysis of thermal behavior of a two-node model for small satellites in Low Earth Orbit", International Journal of Mechanical Sciences, 133: 513-523, (2017).

[18] Farrahi, A., Pérez-Grande, I., "Simplified analysis of the thermal behavior of a spinning satellite flying over Sun-synchronous orbits", Applied Thermal Engineering, 125: 1146-1156, (2017).

[19] Pérez-Grande, I., Sanz-Andrés, A., Guerra, C., Alonso, G., "Analytical study of the thermal behaviour and stability of a small satellite", Applied Thermal Engineering, 29: 2567-2573, (2009). 
[20] Gaite, J., Sanz-Andrés, A., Pérez-Grande, I., "Nonlinear analysis of a simple model of temperature evolution in a satellite", Nonlinear Dynamics, 58: 405, (2009).

[21] Gaite, J., "Nonlinear analysis of spacecraft thermal models", Nonlinear Dynamics, 65: 283-300, (2011).

[22] Baturkin, V., "Micro-satellites thermal control—concepts and components", Acta Astronautica, 56:161-170, (2005).

[23] Das, T. K., Totani, T., Wakita, M., Nagata, H., "A simple thermal design procedure for micro-and nano-satellites with deployable solar array panel", 45th International Conference on Environmental Systems, Bellevue, Washington, USA, (2015).

[24] Bulut, M., Sozbir, N., "Analytical investigation of a nanosatellite panel surface temperatures for different altitudes and panel combinations", Applied Thermal Engineering, 75: 1076-1083, (2015).

[25] Krishnaprakas, C.K., "A comparison of ODE solution methods for spacecraft thermal problems", Heat Transfer Engineering, 19: 103-109, (1998).

[26] Lyon, R., Sellers, J., Underwood, C., "Small satellite thermal modeling and design at USAFA: FalconSat-2 applications", Proceedings IEEE Aerospace Conference, Big Sky, MT, USA, 7-7, (2002).

[27] Diaz-Aguado, M., Greenbaum, J., Fowler, W., Lightsey, G., "Small satellite thermal design, test, and analysis", Orlando, FL, USA, (2006).

[28] Badari Narayana, K., Venkata Reddy, V., "Thermal design and performance of HAMSAT", Acta Astronautica, 60: 7-16, (2007).

[29] Sozbir, N., Bulut, M., Oktem, M., Kahriman, A., Chaix, A., "Design of thermal control subsystem for TUSAT telecommunication satellite", International Journal of Electrical, Computer, Energetic, Electronic and Communication Engineering, 2: 1384-1387, (2008).

[30] Bulut, M., Kahriman, A., Sözbir, N., "Design and analysis for thermal control system of nanosatellite", Vancouver, British Columbia, Canada, (2010).

[31] Dinh, D., "Thermal modeling of nanosat", MSc Thesis, San Jose State University California, USA, (2012).

[32] Garzon, M. M., "Development and analysis of the thermal design for the OSIRIS-3U cubesat", Master's Thesis, Pennsylvania State University Aerospace Engineering, State College, Pennsylvania, USA, 91, (2012).

[33] Athirah, N., Afendi, M., Hafizan, K., Amin, N. A. M., Majid, M. S. A., "Stress and thermal analysis of cubesat structure", Applied Mechanics and Materials, 554: 426-430, (2014).

[34] Silva, D. F. D., Muraoka, I., Garcia, E. C., "Thermal control design conception of the Amazonia-1 satellite", Journal of Aerospace Technology and Management, 6: 169-176, (2014).

[35] Wachche, S., Marne, A., Singare, S., Naik, P., Bhide, O., Chaudhari G., Vartak P., Pendse S., Tadwalkar C., "Thermal modelling and simulation of a pico-satellite using finite element method", 5th International Conference on Thermal Process Modeling and Computer Simulation, Orlando, FL, USA, (2014). 
[36] Flecht, T., "Thermal modelling of the PICSAT nanosatellite platform and synergetic prestudies of the CIRCUS nanosatellite", Master's Thesis, Luleå University of Technology Department of Computer Science, Electrical and Space Engineering, Paris, France, (2016).

[37] Chandrashekar, S., "Thermal analysis and control of MIST cubesat", Master, KTH Royal Institute of Technology Department of Space and Plasma Physics, Stockholm, Sweden, 85, (2017).

[38] Björnberg, A., Larsson, E., "Thermal analysis and control of MIST", KTH Royal Institute of Technology Department of Space and Plasma Physics, Stockholm, Sweden, (2017).

[39] Lahrichi, A., "Heat transfer modeling and simulation of MaSat-1", Al Akhawayn University School of Science and Engineering, Ifran, Morocco, 44, (2017).

[40] Still, V., "Thermal Control Design and Simulation of a Space Mission”, Master's Thesis, Luleå University of Technology Department of Computer Science, Electrical and Space Engineering, 83, (2018).

[41] Wallace, P., Kalapura, A., Kim, S. I., "Thermal modelling and analysis of a cube satellite, EIRSAT-1: steady analysis”, Orlando, FL, USA, (2018).

[42] Deiml, M., Suderland, M., Reiss, P., Czupalla, M., "Development and evaluation of thermal model reduction algorithms for spacecraft", Acta Astronautica, 110: 168-179, (2015).

[43] Corpino, S., Caldera, M., Nichele, F., Masoero, M., Viola, N., "Thermal design and analysis of a nanosatellite in low earth orbit", Acta Astronautica, 115: 247-261, (2015).

[44] Anh, N.D., Hieu, N.N., Chung, P.N., Anh, N.T., "Thermal radiation analysis for small satellites with single-node model using techniques of equivalent linearization", Applied Thermal Engineering, 94: 607-614, (2016).

[45] Bulut, M., Sözbir, Ö., Sözbir, N., "Thermal control of Turksat 3U nanosatellite", 5th International Symposium on Innovative Technologies in Engineering and Science, Baku, Azerbaijan, 26-32, (2017).

[46] Steven, H., Huzain, M. F., "Requirements and design structure for Surya Satellite-1", IOP Conference Series: Earth and Environmental Science, Bogor, Indonesia, 149: 1-10, (2018).

[47] Kovács, R., Józsa, V., "Thermal analysis of the SMOG-1 PocketQube satellite”, Applied Thermal Engineering, 139: 506-513, (2018).

[48] Bonnici, M., Mollicone, P., Fenech, M., Azzopardi, M.A., "Analytical and numerical models for thermal related design of a new pico-satellite", Applied Thermal Engineering, 159: 113908, (2019).

[49] Elhefnawy, A., Elweteedy, A., "Thermal Analysis of a Small Satellite in Post-Mission Phase”, Journal of Multidisciplinary Engineering Science and Technology (JMEST), 6: 10, (2019).

[50] Peabody, H., "Use of TSS as a neutral format for geometry model conversions: An alternative to STEPTAS", 16th European Workshop on Thermal and ECLS Software, Noordwijk, The Netherlands, (2002).

[51] Panczak, T., Rickman, S., Fried, L., Welch, M., “Thermal Synthesizer System: An integrated approach to spacecraft thermal analysis", SAE Transactions, 100: 1851-1867, (1991).

[52] Planas Almazan, P., Flett, D., "ESARAD: From R\&D to industrial utilization”, ESA bulletin, 61-67, (1998). 
[53] Yang, K., Server, N. T. R., "The anatomy of ESATAN and ESARAD thermal model files”, Pasadena, California, USA, (2013).

[54] Duffy, K., "Intorduction to NX TMG Space Thermal", 20th European Workshop on Thermal and ECLS Software, Noordwijk, The Netherlands, (2006).

\section{Nomenclature}

$\begin{array}{ll}a & \text { planetary Albedo coefficient } \\ A & \text { area } \\ \mathrm{C} & \text { specific heat capacity } \\ \mathrm{C} & \text { thermal capacitance } \\ F & \text { view factor } \\ k & \text { thermal conductivity } \\ K & \text { conductive couplings } \\ L & \text { length } \\ q & \text { heat flux per unit area } \\ Q & \text { heat rate } \\ R & \text { radiative couplings } \\ \mathrm{T} & \text { temperature } \\ \alpha & \text { absorptance } \\ \varepsilon & \text { emmisitivity } \\ \phi & \text { solar zenith angle } \\ \rho & \text { density } \\ \sigma & \text { Stefan-Boltzmann constant } \\ \theta & \text { solar ray angle }\end{array}$

\section{Subscript}

$\begin{array}{ll}\text { Albedo } & \text { Albedo } \\ c & \text { cold } \\ h & \text { hot } \\ \text { in } & \text { inlet } \\ i & \text { specified node number } \\ j & \text { specified node number } \\ \text { out } & \text { outlet } \\ \text { planet } & \text { planet } \\ \text { radiated } & \text { radiated } \\ \text { solar } & \text { solar } \\ s / c-p & \text { spacecraft-planet } \\ x & \text { x-direction } \\ y & \text { y-direction } \\ z & \text { z-direction }\end{array}$

